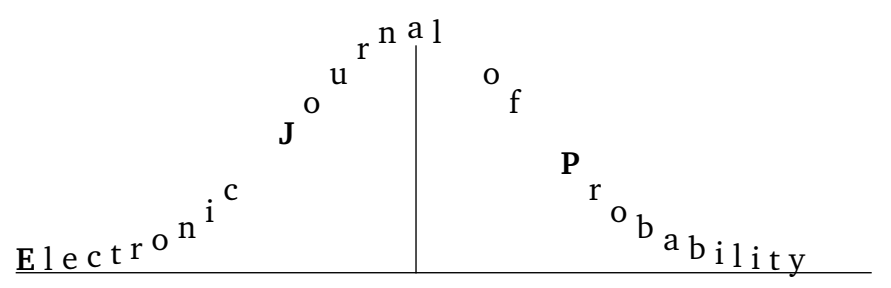

Vol. 15 (2010), Paper no. 66, pages 2019-2040.

Journal URL

http://www.math.washington.edu/ ejpecp/

\title{
Trees, Animals, and Percolation on Hyperbolic Lattices
}

\author{
Neal Madras* and C. Chris $\mathrm{Wu}^{\dagger}$
}

\begin{abstract}
We study lattice trees, lattice animals, and percolation on non-Euclidean lattices that correspond to regular tessellations of two- and three-dimensional hyperbolic space. We prove that critical exponents of these models take on their mean field values. Our methods are mainly combinatorial and geometric.
\end{abstract}

Key words: Percolation, lattice animal, lattice tree, critical exponents, mean field behaviour, hyperbolic geometry, hyperbolic lattice.

AMS 2000 Subject Classification: Primary 60K35; Secondary: 05B45, 51M09, 82B41, 82B4.

Submitted to EJP on July 24, 2009, final version accepted September 7, 2010.

\footnotetext{
${ }^{*}$ Department of Mathematics and Statistics, York University, 4700 Keele Street, Toronto, Ontario M3J 1P3 Canada; madras@mathstat . yorku . ca. Research supported in part by NSERC of Canada.

'Department of Mathematics, Penn State University, Beaver Campus, 100 University Drive, Monaca, PA 15061, USA; ccw3@psu . edu. Research supported in part by NSF grant DMS-05-05484.
} 


\section{Introduction}

Although discrete models of statistical mechanics are usually based on Euclidean lattices such as $\mathbb{Z}^{d}$ (the $d$-dimensional hypercubic lattice), some researchers have studied the properties of standard models on various non-Euclidean lattices. See for example Grimmett and Newman (1990), Rietman et al. (1992), Swierczak and Guttmann (1996), Benjamini and Schramm (1996, 2001), Wu (2000), Lalley (1998, 2001), Benjamini et al. (1999), Jonasson and Steif (1999), Pak and Smirnova-Nagnibeda (2000), Schonmann (2001, 2002), Madras and Wu (2005), Häggström and Jonasson (2006), and Tykesson (2007).

In this paper we shall study properties of three standard statistical mechanical models on hyperbolic lattices. Hyperbolic lattices differ from Euclidean lattices in that the number of sites within distance $N$ of the origin grows exponentially in $N$ rather than polynomially. In this sense hyperbolic lattices are like infinite regular trees, but unlike trees they have cycles.

The models from statistical mechanics that we study here are lattice animals (finite connected subgraphs of the lattice), lattice trees (lattice animals with no cycles), and percolation (a random graph model in which bonds of the lattice are randomly "occupied" or "vacant"). Lattice animals and lattice trees model branched polymers (see Vanderzande (1998) or Janse van Rensburg (2000)), while percolation models a random medium (see Grimmett (1999)). We shall prove that these three models all exhibit "mean field" scaling behaviour on hyperbolic lattices in two and three dimensions. We expect that this is true in higher dimensional hyperbolic lattices as well, but, as we shall discuss, our method falls slightly short of proving this.

The paper is organized as follows. This introductory section contains three subsections. Subsection 1.1 introduces the hyperbolic lattices on which we shall work. Subsection 1.2 introduces lattice animals and lattice trees and presents our main results about them. Subsection 1.3 introduces percolation and presents our main result about it. Section 2 presents basic definitions and properties about hyperbolic lattices and hyperbolic geometry that we will need in subsequent sections. Section 3 proves the basic result that lattice animals and lattice trees grow at a well-defined exponential rate in the number of sites. The proof requires a fair number of intermediate results and definitions. Although occasionally cumbersome, the work in Section 3 pays big dividends in Sections 4 and 5 , where it leads to relatively direct proofs of the main scaling results about lattice animals (and trees) and percolation respectively.

Notation: If $u$ is a real number, then $\lfloor u\rfloor$ denotes the greatest integer less than or equal to $u$. If $S$ is a set, then $|S|$ denotes the cardinality of $S$. For functions $f$ and $g$ defined on a set $S$, we write $f \asymp g$ to mean that there exist positive finite constants $K_{1}$ and $K_{2}$ such that $K_{1} f(z) \leq g(z) \leq K_{2} f(z)$ for all $z \in S$. And if $z_{0}$ is a specified limit point of $S$ (possibly $\infty$ ), then we write $f \sim g$ to mean that $f(z) / g(z)$ converges to 1 as $z \rightarrow z_{0}$.

\subsection{Hyperbolic Lattices}

In this paper we shall explicitly consider specific two- and three-dimensional hyperbolic lattices (see Section 2 for explicit definitions of this and other terms), although our general approach should work for other hyperbolic lattices. For that reason we keep our terminology general in places. There is one place where our argument does not seem to hold above three dimensions (see the remark 
following Lemma 8), but we believe that there is a "better" argument that will allow the rest of our work to extend (in principle) above three dimensions.

Our two-dimensional lattices are derived from regular tessellations (tilings) of the hyperbolic plane $\mathbb{H}^{2}$. These tessellations are characterized by two integers $f$ and $v$, both greater than 2 , such that $(f-2)(v-2)>4$. The integer $f$ is the number of sides (or vertices) on each polygonal tile, and $v$ is the number of tiles that meet at each vertex. The "Schläfli symbol" for this tessellation is $\{f, v\}$ (Coxeter 1963). We shall denote the graph consisting of the edges and vertices in the tessellation as $\mathscr{H}(f, v)$ (see Figure 1). (In Madras and Wu (2005), we used $\mathscr{H}(v, f)$, but in the present paper we conform to the standard ordering.)

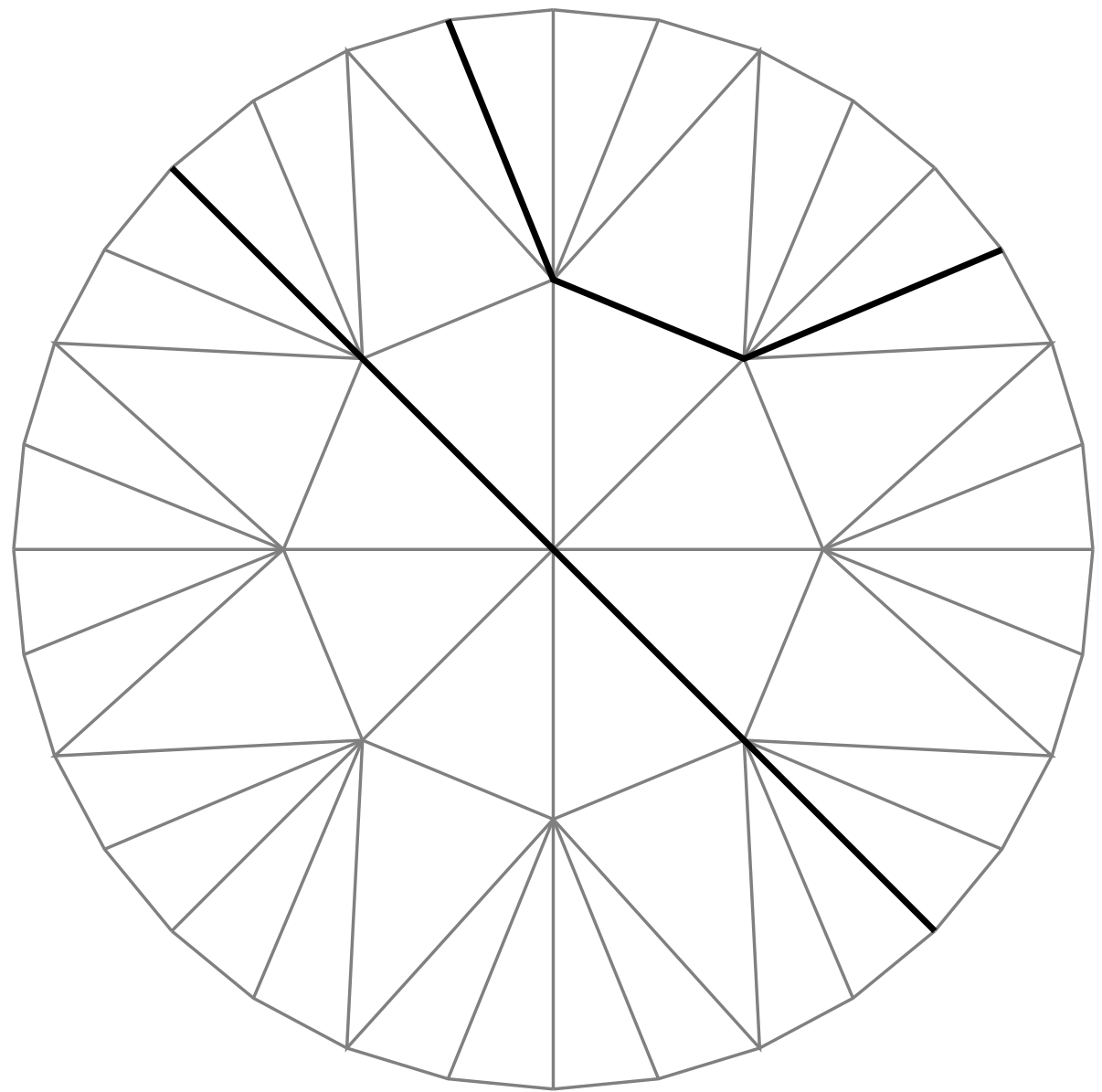

Figure 1: Part of the hyperbolic graph $\mathscr{H}(3,8)$. The two heavy lines correspond to two examples of lattice-lines (see Section 2).

Our three-dimensional lattice is derived from the regular tessellation of hyperbolic three-space $\mathbb{H}^{3}$ by dodecahedra, with all dihedral angles equal to $\pi / 2$. Thus eight dodecahedra meet at each vertex, and four dodecahedra share each edge. (The "Schläfli symbol" for the dodecahedron is $\{5,3\}$, since 3 pentagons meet at each vertex. The Schläfli symbol for this tessellation is $\{5,3,4\}$. See Coxeter $(1956,1963)$.) We shall denote the graph consisting of the edges and vertices in this tessellation by $\mathscr{H}(5,3,4)$.

In the $\{5,3,4\}$ tessellation, consider an arbitrary facet of some dodecahedron tile, and consider the 
plane in $\mathbb{H}^{3}$ that contains this facet. Because the facets of each dodecahedron meet at right angles, this plane is the union of infinitely many facets of dodecahedra. Indeed, these facets form a $\{5,4\}$ tessellation of the given plane (four pentagons meeting at each vertex). Such a plane is called a "lattice-plane" below (see Section 2 for the formal definition). The existence of these lattice-planes make it easy to work with the lattice $\mathscr{H}(5,3,4)$; for example, every edge that intersects the latticeplane has at least one endpoint in the lattice-plane. For convenience, we shall also work with two-dimensional lattices that have an analogous property: namely, the line in $\mathbb{H}^{2}$ that contains a given edge of $\{f, v\}$ should be the union of edges of $\{f, v\}$. This is true if and only if $v$ is even (see Section 3 of Madras and Wu (2005) for more discussion). Henceforth we shall assume that $v$ is even in $\mathscr{H}(f, v)$.

We close this subsection with remarks on other regular hyperbolic lattices; see Coxeter (1956) for a fuller survey. There are three other regular tessellations of $\mathscr{H}^{3}$ by bounded tiles: $\{3,5,3\}$ has icosahedra with dihedral angles $2 \pi / 3 ;\{4,3,5\}$ has "cubes" with dihedral angles $2 \pi / 5$; and $\{5,3,5\}$ has dodecahedra with dihedral angles $2 \pi / 5$. There are five regular tessellations of $\mathscr{H}^{4}$ with bounded tiles, but there are none of $\mathscr{H}^{d}$ for any $d>4$. We do not know if any of these other tessellations has a nice "lattice-(hyper)plane" structure similar to that of $\{5,3,4\}$.

Of course, one could weaken the regularity assumption and consider tessellations of hyperbolic space in which the tiles are polytopes but not regular. In this case, we would like the union of all facets of all tiles to be equal to a (countable) union of hyperplanes. (This condition has been studied in $\mathbb{H}^{2}$ by Bowen and Series (1992) and in higher dimensions by Bourdon (1993), among others.) We fully expect our methods to extend to all such tessellations of $\mathbb{H}^{2}$ and $\mathbb{H}^{3}$, although it seems cumbersome to give our proofs in such generality (analogously, authors often work only on the lattices $\mathbb{Z}^{d}$ rather than on general Euclidean lattices).

\subsection{Lattice Animals and Lattice Trees}

In this paper we shall use $\mathscr{G}$ to denote one of the "lattices" that we are considering: the hyperbolic lattices $\mathscr{H}(f, v)$ and $\mathscr{H}(5,3,4)$, and sometimes the Euclidean lattices $\mathbb{Z}^{d}$. One site of the lattice $\mathscr{G}$ is chosen to be called the "origin".

A lattice animal is a finite connected subgraph of $\mathscr{G}$. A lattice tree is a lattice animal with no cycles.

For each integer $N \geq 1$, let $a_{N}$ (respectively, $t_{N}$ ) denote the number of lattice animals (respectively, trees) that have exactly $N$ sites and contain the origin. Also, for each lattice site $x$ of $\mathscr{G}$, let $a_{N}(x)$ (respectively, $t_{N}(x)$ ) denote the number of lattice animals (respectively, trees) that have exactly $N$ sites and contain both $x$ and the origin.

Remark: In the case of the $d$-dimensional integer lattice $\mathbb{Z}^{d}$ in Euclidean space, one often works with $a_{n}^{(0)}$, the number of $n$-vertex lattice animals modulo translation. (That is, if we say that two animals are equivalent when one is a translation of the other, then $a_{n}^{(0)}$ is the number of equivalence classes of animals having $n$ vertices.) Then $a_{n}^{(0)}=a_{n} / n$. There is no analogous simple equation if we replace "translation" by "automorphism", since some animals are mapped onto themselves by a non-trivial automorphism. Since translation in non-Euclidean geometry is not as well behaved as Euclidean translation, we shall not define an analogue of $a_{n}^{(0)}$ for hyperbolic graphs (although $\mathscr{A} \mathscr{L}_{n}$ in Definition 6 will come closest).

The following basic result extends what we know from Euclidean lattices (Klarner, 1967; Klein, 1981; Janse van Rensburg, 2000) to our hyperbolic graphs. We shall prove it in Section 3 , 
Proposition 1. Let $\mathscr{G}$ be one of the hyperbolic lattices $\mathscr{H}(f, v)$ or $\mathscr{H}(5,3,4)$. Then there exist positive finite constants $\lambda_{a}$ and $\lambda_{t}$ (depending on the lattice) such that

$$
\lambda_{a}=\lim _{N \rightarrow \infty} a_{N}^{1 / N} \text { and } \quad \lambda_{t}=\lim _{N \rightarrow \infty} t_{N}^{1 / N} .
$$

Moreover, there exist positive finite constants $B_{a}$ and $B_{t}$ such that

$$
a_{N} \leq B_{a} \lambda_{a}^{N} \quad \text { and } \quad t_{N} \leq B_{t} \lambda_{t}^{N} \quad \text { for all } N \geq 1
$$

The number $\lambda_{a}$ (respectively $\lambda_{t}$ ) is called the growth constant for lattice animals (respectively trees) on the given lattice.

It is generally believed that the numbers of lattice animals and lattice trees have the following asymptotic behaviour:

$$
a_{n} \sim K_{a} n^{-\theta_{a}} \lambda_{a}^{n} \quad \text { and } \quad t_{n} \sim K_{t} n^{-\theta_{t}} \lambda_{t}^{n} \quad \text { as } n \rightarrow \infty
$$

In Euclidean lattices, the relations of (3) are more commonly written in terms of $a_{n}^{(0)}\left(=a_{n} / n\right)$ and $t_{n}^{(0)}\left(=t_{n} / n\right)$, viz.

$$
a_{n}^{(0)} \sim K_{a} n^{-\theta_{a}^{(0)}} \lambda_{a}^{n} \quad \text { and } \quad t_{n}^{(0)} \sim K_{t} n^{-\theta_{t}^{(0)}} \lambda_{t}^{n} \quad \text { as } n \rightarrow \infty
$$

with $\theta_{a}^{(0)}=\theta_{a}+1$ and $\theta_{t}^{(0)}=\theta_{t}+1$. The constants $K_{a}, K_{t}, \lambda_{a}$, and $\lambda_{t}$ depend on the lattice, but it is believed that the critical exponents $\theta_{a}$ and $\theta_{t}$ depend only on the ambient space. Moreover, lattice animals and lattice trees are believed to be in the same universality class (Lubensky and Isaacson, 1979), which would imply that $\theta_{a}=\theta_{t}$ for every lattice. The common value of $\theta_{a}^{(0)}$ and $\theta_{t}^{(0)}$ is believed to equal 1 in any two-dimensional Euclidean lattice, and $3 / 2$ in any threedimensional Euclidean lattice (Parisi and Sourlas, 1981; Brydges and Imbrie, 2003). The common value in $d$-dimensional Euclidean space is believed to increase steadily with $d$, until $d=8$. Lace expansion methods have been used to prove that $\theta_{a}^{(0)}=\theta_{t}^{(0)}=5 / 2$ for $\mathbb{Z}^{d}$ with sufficiently large $d$, as well as for specific Euclidean lattices in every dimension above 8 (see Slade (2006) and the references therein). Madras (1995) proved that $\theta_{a}^{(0)}$ and $\theta_{t}^{(0)}$ are bounded below by $(d-1) / d$ in any $d$-dimensional Euclidean lattice (in the sense that $a_{n}^{(0)} \leq C_{a} n^{-(d-1) / d} \lambda_{a}^{n}$ for all $n$ and a constant $C_{a}$, and similarly for $\left.t_{n}^{(0)}\right)$. It is also known that $\theta_{a}^{(0)}$ and $\theta_{t}^{(0)}$ are bounded above by $5 / 2$ on very general lattices (Bovier et al., 1986; Tasaki and Hara, 1987), in the sense of a proven bound on the generating functions; see Equations (19) and (5).

The value $\theta^{(0)}=5 / 2$, or equivalently $\theta=3 / 2$, has appeared over the years in many similar problems of asymptotic enumeration of trees. An early landmark paper was Pólya (1937) (see Pólya and Read (1987) for translation and discussion), which examined the asymptotic enumeration of classes of "abstract" graphs (in contrast to lattice animals, which are embeddings of graphs into a lattice in a geometric space). For example, consider the number of topologically different trees with $n$ vertices of degree 4 and all other vertices of degree 1 (the "degree" of a vertex is the number of its neighbours); this counts the number of possible structurally different molecules (isomers) with the formula $\mathrm{C}_{n} \mathrm{H}_{2 n+2}$. Polya showed that this sequence has asymptotic behaviour of the form $K n^{-3 / 2} \tau^{n}$. Otter (1948) proved the same asymptotic form for the number of trees having $n$ vertices and maximum degree $m$ (fixed). This result also arises in the context of percolation on a tree: 
Equation (10.12) in Grimmett (1999) implies that the rooted infinite binary tree has $\left(\begin{array}{c}2 n \\ n-1\end{array}\right) / n \sim$ $K n^{-3 / 2} 4^{n} n$-node subtrees containing the root. In many ways, abstract trees are easier to deal with than lattice trees, since the former need not deal with spatial interaction in the form of mutual avoidance of branches. For this reason, we sometimes refer to abstract trees as a mean field model for lattice trees, and we refer to $3 / 2$ as the mean field value of the critical exponent $\theta$ for trees (or animals). (See Borgs et al. (1999) for a different mean field model of lattice trees, in which trees are embedded in the lattice $\mathbb{Z}^{d}$ with self-intersections permitted.) Lattice trees share the following property with many other important models of statistical mechanics in Euclidean space: there is an "upper critical dimension" above which the critical exponents take on their mean field values. For lattice trees and animals, the upper critical dimension is 8 . In hyperbolic lattices, in contrast, we shall prove that the mean field value $\theta=3 / 2$ occurs in 2 and 3 dimensions (and we expect it to hold in every dimension above 1 ).

We shall prove that $\theta=3 / 2$ in the context of generating functions, which is slightly weaker than Equation (3). To this end, we now define generating functions for trees and animals, as functions of a continuous variable $z$. In general, we permit $z$ to be complex, but in the present paper we shall only deal with real positive values of $z$.

Definition 2. In the following, $z$ is a complex variable and $x$ is a vertex. The two-point functions are

$$
\begin{aligned}
G_{a}(x ; z) & :=\sum_{N=1}^{\infty} a_{N}(x) z^{N}, \\
G_{t}(x ; z) & :=\sum_{N=1}^{\infty} t_{N}(x) z^{N} \quad \text { for } x \in V(\mathscr{G}) .
\end{aligned}
$$

The susceptibility functions are

$$
\begin{aligned}
& \chi_{a}(z):=\sum_{x \in V} G_{a}(x ; z)=\sum_{N=1}^{\infty} N a_{N} z^{N}, \\
& \chi_{t}(z):=\sum_{x \in V} G_{t}(x: z)=\sum_{N=1}^{\infty} N t_{N} z^{N} .
\end{aligned}
$$

Finally, denote the radii of convergence of $\chi_{a}$ and $\chi_{t}$ respectively as

$$
z_{a c}:=\frac{1}{\lambda_{a}} \quad \text { and } \quad z_{t c}:=\frac{1}{\lambda_{t}}
$$

Observe that if the scaling behaviour (3) holds, then we also have

$$
\chi_{a}(z) \sim \tilde{K}_{a}\left(z_{a c}-z\right)^{\theta_{a}-2} \text { as } z \nearrow z_{a c}=\lambda_{a},
$$

if $\theta_{a}<2$ (with an analogous assertion for $\chi_{t}$ ).

In Section 4 we shall prove the following result, which implies that if Equation (3) is true in our hyperbolic lattices, then $\theta_{a}=\theta_{t}=3 / 2$.

Theorem 3. For the hyperbolic lattices $\mathscr{H}(f, v)$ in $\mathbb{H}^{2}$ and $\mathscr{H}(5,3,4)$ in $\mathbb{H}^{3}$, we have

$$
\chi_{a}(z) \asymp\left(z_{a c}-z\right)^{-1 / 2}
$$

for all $z$ in $\left(z_{a c} / 2, z_{a c}\right)$. The corresponding assertion for trees also holds. 


\subsection{Percolation}

We shall focus on bond percolation here, although changes needed for site percolation seem minimal.

Consider a lattice $\mathscr{G}$ and a parameter $p \in[0,1]$. For each bond $e$ of $\mathscr{G}$, let $B_{e}$ be an independent Bernoulli random variable that equals 1 (respectively 0 ) with probability $p$ (respectively $1-p$ ). We say that $e$ is occupied if $B_{e}=1$, and vacant if $B_{e}=0$. The set of occupied edges induces a subgraph of $\mathscr{G}$, and the connected components of this random subgraph are called the percolation clusters. The interesting objects in this model are the large clusters; in particular, can clusters be infinite?

We write $P_{p}$ to denote the probability measure of the above model (i.e. of the collection of all $B_{e}$ 's), and $E_{p}$ to denote the corresponding expectation. For a vertex $x \in V$, we write $\mathscr{C}(x)$ to denote the percolation cluster containing $x$. A fundamental quantity is the function

$$
\theta(p):=P_{p}\{|\mathscr{C}(0)|=\infty\}
$$

the probability that the origin is connected to infinitely many sites via paths of open bonds. We define the critical probability $p_{c}$ by

$$
p_{c}:=\inf \{p \in[0,1]: \theta(p)>0\} .
$$

Standard arguments (see for example Section 1.4 of Grimmett (1999)) show that $0<p_{c}<1$ for hyperbolic lattices (as well as many other lattices). In addition, we define the percolation susceptibility function

$$
\chi(p):=E_{p}(|\mathscr{C}(0)|)
$$

and, for $q \in(0,1)$, the function

$$
\theta(p, q):=E_{p}\left(1-(1-q)^{|\mathscr{C}(0)|}\right) .
$$

It is of major interest to understand the scaling properties of the above functions for $p$ near $p_{c}$. The scaling behaviours are described by critical exponents $\gamma, \Delta, \delta$, and $\beta$, among others (see Grimmett (1999) for a full discussion). The critical exponent $\gamma$ may be defined by the property $\chi(p) \sim$ $\left(p_{c}-p\right)^{-\gamma}$ as $p \uparrow p_{c}$; we shall use the slightly weaker definition

$$
\chi(p) \asymp\left(p_{c}-p\right)^{-\gamma} \quad \text { for } p \in\left(0, p_{c}\right) .
$$

In particular, the assertion " $\gamma=1$ " means "the scaling relation (8) holds with $\gamma=1$ ". Similarly, we define $\beta, \delta$, and $\Delta$ by

$$
\begin{gathered}
\theta(p) \asymp\left(p_{c}-p\right)^{\beta} \quad \text { for } p \in\left(p_{c}, 1\right) ; \\
\theta\left(p_{c}, q\right) \asymp q^{1 / \delta} \quad \text { for } 0<q<1 ; \text { and } \\
E_{p}\left(|\mathscr{C}(0)|^{m+1}\right) / E_{p}\left(|\mathscr{C}(0)|^{m}\right) \asymp\left(p_{c}-p\right)^{\Delta} \quad \text { for } p \in\left(0, p_{c}\right), m=1,2, \ldots
\end{gathered}
$$

Our results about scaling behaviour of percolation are contained in the following result, which we shall prove in Section 5. It says that percolation in our hyperbolic lattices takes on its mean field values (i.e., values corresponding to percolation on a tree-see Theorem 10.52 of Grimmett (1999)). In contrast, percolation in Euclidean lattices only takes on mean field values above 6 dimensions (see Slade (2006) for discussion and references). 
Theorem 4. For the hyperbolic lattices $\mathscr{H}(f, v)$ in $\mathbb{H}^{2}$ and $\mathscr{H}(5,3,4)$ in $\mathbb{H}^{3}$, we have $\gamma=1, \beta=1$, $\delta=2$, and $\Delta=2$.

Schonmann (2002) proved this result for percolation on a large class of planar graphs that includes our lattices $\mathscr{H}(f, v)$, but his methods relied heavily on planarity. In contrast, our methods apply equally to two- and three-dimensional hyperbolic lattices, and may well apply in four or more dimensions. However, our methods make substantial use of hyperbolic geometry, and it is not clear whether they will extend to general non-amenable graphs that do not have a nice embedding in hyperbolic space.

Our percolation proofs are very geometric and combinatorial, and focus on sets of lattice animals. In a sense, we treat percolation functions like certain kinds of generating functions of lattice animals. Indeed, it is interesting to note that our percolation proofs do not use any correlation inequalities (such as FKG or BK-see Grimmett (1999)). Rather, they are modelled closely on the lattice animal proofs of Section 4 .

\section{Definitions and Basic Hyperbolic Geometry}

Let $S$ be a subset of $\mathbb{H}^{d}$ or $\mathbb{R}^{d}$. The convex hull of $S$, denoted $\operatorname{conv}(S)$, is the intersection of all closed half-spaces containing $S$. We say that a polytope is the convex hull of a finite set of points (elsewhere, this is often called a bounded convex polytope). Aăface of a convex set $C$ is a convex subset $F$ of $C$ with the following property: if a line segment in $C$ has an interior point in $F$, then the line segment must be entirely contained in $F$. A zero-dimensional face is called an extreme point; a one-dimensional face is called an edge; and a $(d-1)$-dimensional face of a $d$-dimensional convex set is called a facet.

A tessellation is a covering of space by a countable collection of polytopes with pairwise disjoint interiors, with all polytopes congruent to one another. We shall use the term tile to refer to any one of the polytopes in a given tessellation. In particular, each tile is a closed set. We shall focus on regular tessellations, in which each tile is a regular polytope and any non-empty intersection of a pair of tiles is a face (of some dimensionality) of both tiles. We define a hyperbolic lattice corresponding to a given tessellation to be the infinite graph whose edges and vertices are the edges and extreme points of the tiles of the tessellation.

Although some of our discussion will be in terms of hyperbolic space in general dimensions, we will mostly focus on the tessellations $\{f, v\}$ in $\mathbb{H}^{2}$ and $\{5,3,4\}$ in $\mathbb{H}^{3}$, as described in Section 1.1, and their associated lattices $\mathscr{H}(f, v)$ and $\mathscr{H}(5,3,4)$.

We write $d_{H}(x, y)$ to denote the usual hyperbolic distance between the points $x$ and $y$ in $\mathbb{H}^{d}$ (Iversen 1992, Ratcliffe 1994). There is a unique possible value $\lambda_{e}$ for the side length (as measured by $d_{H}$ ) of a regular pentagon with five right angles (in fact, $\lambda_{e} \approx 1.0612$ because $\lambda_{e}$ is the positive root of $\sinh ^{2} \lambda_{e}=\cosh \lambda_{e}$, which equals $\lambda_{e}=\ln (\tau+\sqrt{\tau})$ where $\tau=(1+\sqrt{5}) / 2$; this may be deduced from p. 112 of Iversen (1992) or p. 97 of Ratcliffe (1994)). If we view the graph $\mathscr{H}(5,3,4)$ as being embedded in $\mathbb{H}^{3}$, so that each edge of the graph corresponds to an edge of a dodecahedron in the tessellation $\{5,3,4\}$, then $\lambda_{e}$ is the hyperbolic length of each edge.

A "lattice-hyperplane" is a hyperplane in $\mathbb{H}^{d}$ which contains a facet of some tile in the tessellation. (These are also called "lattice-lines" in $\mathbb{H}^{2}$ and "lattice-planes" in $\mathbb{H}^{3}$. See Figure 1).) In the tessellations we consider $(\{f, v\}$ with even $v$, and $\{5,3,4\})$, every lattice-hyperplane is the union of an 
infinite collection of facets in the tessellation. A "lattice-half-space" is a half-space whose boundary is a lattice-hyperplane. (We will be careful not to omit the "lattice" prefix when it is needed, since the possibility of confusion is quite real.)

We denote the set of vertices of the lattice $\mathscr{G}$ by $V(\mathscr{G})$, or simply $V$. Fix one of the vertices of $\mathscr{G}$ to be the origin, and denote it 0 . The graph distance between two vertices $u$ and $v$ of $\mathscr{G}$ is defined to be the fewest number of edges on a path in $\mathscr{G}$ from $u$ to $v$, and we denote it $d_{G}(u, v)$. If $v \in V$ and $A \subset \mathbb{H}^{d}$, then $d_{G}(v, A)$ is the minimum value of $d_{G}(v, w)$ among all $w \in V \cap A$.

We denote the cardinality of a set $S$ by $|S|$. We shall extend this notation to the situation that $S$ is a subgraph of $\mathscr{G}$, in which case $|S|$ shall denote the number of vertices in $S$.

Let $S$ be any subset of $\mathbb{H}^{d}$. For a given tessellation, the neighborhood of $S$, denoted $\mathscr{N}(S)$, is the union of all tiles that have nonempty intersection with $S$.

We shall frequently refer to automorphisms of the graph $\mathscr{G}$. A (graph) automorphism is a bijection of $V(\mathscr{G})$ with itself that preserves adjacency; in particular, an automorphism extends naturally to a bijection on the set of edges. Also, we can view each automorphism of $\mathscr{G}$ as the restriction to $V(\mathscr{G})$ of a particular isometry of $\mathbb{H}^{d}$. We shall write $K^{\prime}$ for the number of automorphisms $\phi$ such that $\phi(0)=0$. It follows that for any two vertices $x$ and $y$, there are exactly $K^{\prime}$ automorphisms of $\mathscr{G}$ such that $\phi(x)=y$.

Lemma 5. There exists positive finite constants $K_{G H}$ and $K_{H G}$ with the following property: For all vertices $u$ and $v$ of $V(\mathscr{G})$,

$$
\frac{1}{K_{G H}} d_{G}(u, v) \leq d_{H}(u, v) \leq K_{H G} d_{G}(u, v)
$$

Proof: Clearly we can take $K_{H G}=\lambda_{e}$, the length of an edge of a tile. For the other inequality, observe that the $d_{H}$-distance between any two disjoint tiles is at least $\lambda_{e}$. Let $\alpha$ be the number of tiles that intersect a fixed tile. Then the number of tiles intersecting an arbitrary segment $\overline{u v}$ is at most $\alpha+\alpha\left\lfloor d_{H}(u, v) / \lambda_{e}\right\rfloor$, which is at most $2 \alpha d_{H}(u, v) / \lambda_{e}$ for distinct vertices $u$ and $v$ (because $d_{H}(u, v) \geq \lambda_{e}$ in this case). Since there is a path connecting vertices $u$ and $v$ that only uses edges of $\mathscr{N}(\overline{u v})$, it follows that we can take $K_{G H}$ to be $2 \alpha / \lambda_{e}$ times the number of edges on a single tile.

\section{Basic Properties of Lattice Animals and Lattice Trees}

Our first task is to prove that the limits

$$
\lim _{N \rightarrow \infty} a_{N}^{1 / N} \text { and } \quad \lim _{N \rightarrow \infty} t_{N}^{1 / N}
$$

exist and are finite. The general idea is the same as in Euclidean lattices, but some complications arise due to lack of translation (as alluded to in the Remark of Section 1.2). We shall concentrate on the case of lattice animals. The proofs for lattice trees are essentially the same.

This section requires some hard work and attention to detail, but fortunately this makes for smooth sailing in the rest of the paper. 
Definition 6. Fix a lattice-half-space $\hat{H}_{0}$ that has the origin on its boundary. For each positive integer $N$, define the following sets of lattice animals:

$$
\begin{aligned}
\mathscr{A}_{N}= & \{C: C \text { is an } N \text {-site animal containing } 0\} ; \\
\mathscr{A} \mathscr{E}_{N}= & \{C: C \text { is an } N \text {-site animal, and } \\
& \quad 0 \text { is an extreme point of } \operatorname{conv}(\mathscr{N}(C))\} ; \\
\mathscr{A} \mathscr{L}_{N}= & \left\{C \in \mathscr{A}_{N}: 0 \in C \subset \hat{H}_{0}\right\} .
\end{aligned}
$$

In particular, we have $a_{N}=\left|\mathscr{A}_{N}\right|$ for every $N$.

Proposition 7. (a) There exists a strictly positive real number $\epsilon_{E}$ such that

$$
\epsilon_{E} a_{N} \leq\left|\mathscr{A}_{\mathscr{E}_{N}}\right| \quad \text { for all } N \geq 1
$$

(b) There exist a positive real number $U_{L}$ and a positive integer $r$ such that

$$
\left|\mathscr{A}_{\mathscr{E}_{N}}\right| \leq U_{L}\left|\mathscr{A} \mathscr{L}_{N+r}\right| \leq U_{L} a_{N+r} \quad \text { for all } N \geq 1
$$

Before we can prove Proposition 7, we need two geometric lemmas for $\mathbb{H}^{3}$ that are analogues of results for $\mathbb{H}^{2}$ in Madras and Wu (2005).

Lemma 8. Fix a tessellation in $\mathbb{H}^{3}$ (with bounded tiles). There exist $\epsilon_{V}>0$ and $\epsilon_{E}>0$ with the following property: For every $N \geq 1$ and every subset $S$ of $V$ that contains exactly $N$ vertices, the volume of $\operatorname{conv}(\mathscr{N}(S))$ is at least $\epsilon_{V} N$, and $\operatorname{conv}(\mathscr{N}(S))$ has at least $\epsilon_{E} N$ extreme points.

Proof: Let $n_{\text {Tile }}$ be the number of vertices of one tile (20 for the dodecahedron). The first assertion holds with $\epsilon_{V}=1 / n_{\text {Tile }}$, since $\mathscr{N}(S)$ contains at least $|S| / n_{\text {Tile }}$ tiles.

The second assertion holds because of the following two facts. First, any 3-dimensional polytope with $n$ extreme points can be decomposed into the union of at most $2 n-4$ tetrahedra, as we shall prove in the next paragraph. Secondly, Haagerup and Munkholm (1981) proved that for any dimension $d \geq 2$ there is a constant $V_{d}$ such that no $d$-simplex in $\mathbb{H}^{d}$ has volume greater than $V_{d}$ (a " $d$-simplex" in $\mathbb{H}^{d}$ or $\mathbb{R}^{d}$ is the convex hull of $d+1$ points that do not all lie in a hyperplane). A 3-simplex is a tetrahedron. Therefore, if $|S|=N$ and $\operatorname{conv}(\mathscr{N}(S))$ has $n$ extreme points, then

$$
\epsilon_{V} N \leq \text { volume of } \operatorname{conv}(\mathscr{N}(S)) \leq(2 n-4) V_{3} .
$$

Thus we can take $\epsilon_{E}=\epsilon_{V} /\left(2 V_{3}\right)$.

Finally, we must prove the first fact in the preceding paragraph. Consider a 3-dimensional polytope with $n$ extreme points. Subdivide each polygonal facet of the polytope into triangles (if the polygonal facet has $k$ edges, then it gets subdivided into $k-2$ triangles). Let $e$ (respectively, $f$ ) denote the number of edges (respectively, triangular facets) in the resulting triangulation of the surface of the polytope. Then $3 f=2 e$. Using this in Euler's formula $n-e+f=2$, we find that $f=2 n-4$. Next pick a point $w$ in the polytope's interior, and for each triangular facet, construct a tetrahedron which is the convex hull of that facet and $w$. This gives $2 n-4$ tetrahedra whose union is the whole polytope, and we are done.

Remark: While we believe that Lemma 8 should also be true in 4 or more dimensions, we do not have a proof. The above proof uses the fact that a 3-dimensional polytope with $n$ vertices has at 
most $O(n)$ facets. The analogue is false in $d$ dimensions for $d>3$, where counterexamples are provided by the class of "cyclic polytopes" (see Grünbaum, 2003). For example, in 4 dimensions, there exist polytopes with $n$ vertices and $\left(n^{2}-3 n\right) / 23$-dimensional facets. This seems to be the only real barrier to direct extension of our work to 4 or more dimensions.

The following result is only proven here for $\mathscr{H}(5,3,4)$, but the analogue for other hyperbolic lattices and dimensions should also be true (with "hyperplane" instead of "plane"). The analogue for the planar lattices $\mathscr{H}(f, v)$ was proven in Lemma 3.2 of Madras and Wu (2005).

Lemma 9. There is a constant $D$ with the following property for the lattice $\mathscr{H}(5,3,4):$ Let $P$ be any plane in $\mathbb{H}^{3}$, and let $x \in V$ be a vertex whose distance from $P$ is at least $D$. Then there is a lattice-plane through $x$ that does not intersect $P$.

Proof: Consider a plane $P$ and point $x$ not on $P$. Let $y$ be the closest point of $P$ to $x$. Let $z$ be any other point of $P$, and consider the triangle $x y z$. Write $\theta_{x}, \theta_{y}$, and $\theta_{z}$ for the angles of the triangle. Then the hyperbolic Law of Cosines [p. 108 of Iversen (1992) or p. 87 of Ratcliffe (1994)] says that

$$
\cosh \left(d_{H}(x, y)\right)=\frac{\cos \theta_{x} \cos \theta_{y}+\cos \theta_{z}}{\sin \theta_{x} \sin \theta_{y}}
$$

Since $\theta_{y}=\pi / 2$ by the definition of $y$, and since $0<\theta_{z}<\pi / 2$, it follows that $\cosh \left(d_{H}(x, y)\right)<$ $1 / \sin \theta_{x}$. Therefore, for any plane $P_{i}$ containing $x$ that intersects $P$, the angle $\theta[i]$ between $P_{i}$ and the segment $\overline{x y}$ satisfies

$$
\sin (\theta[i])<\frac{1}{\cosh \left(d_{H}(x, y)\right)}
$$

Now suppose $x \in V$ and let $P_{1}, P_{2}$, and $P_{3}$ be the three lattice planes through $x$. Assume that each $P_{i}$ intersects $P$. Define the angles $\theta[i]$ as above $(i=1,2,3)$. Then

$$
1=\sum_{i=1}^{3} \sin ^{2}(\theta[i])<\frac{3}{\cosh ^{2}\left(d_{H}(x, y)\right)} .
$$

This implies $\cosh \left(d_{H}(x, y)\right)<\sqrt{3}$, i.e. $d_{H}(x, y)<\ln (\sqrt{3}+\sqrt{2})$. Therefore we see that the assertion of the Lemma holds with $D=\ln (\sqrt{3}+\sqrt{2})$.

Proof of Proposition 7: (a) Consider the collection of all 4-tuples $(C, y, \hat{C}, \phi)$ such that $C \in \mathscr{A}_{N}, y$ is an extreme point of $\operatorname{conv}(\mathscr{N}(C)), \hat{C} \in \mathscr{A}_{\mathscr{E}_{N}}$, and $\phi$ is an automorphism of $\mathscr{G}$ such that $\phi(y)=0$ and $\hat{C}=\phi(C)$ [i.e., $\hat{C}$ is the image of $C$ under $\phi$ ]. How many such 4-tuples are there? For each given $\hat{C} \in \mathscr{A} \mathscr{E}_{N}$, there exist $N$ possible choices for the vertex $\phi(0)$, namely the $N$ vertices of $\hat{C}$ [notice that $\phi(0)$ must be in $\hat{C}$ because $0 \in C$ ]. If we know $\hat{C}$ and $\phi(0)$, then there are exactly $K^{\prime}$ possibilities for $\phi$, where $K^{\prime}$ is the number of automorphisms of $\mathscr{G}$ that fix the origin. Since $\phi$ is invertible, the choice of $\hat{C}$ and $\phi$ determines $C$ and $y$. Therefore

$$
\text { (number of 4-tuples containing } \hat{C} \text { ) }=K^{\prime} N \quad \text { for each } \hat{C} \in \mathscr{A}_{\mathscr{E}_{N}} \text {, }
$$

which implies that

$$
\text { (number of 4-tuples) }=K^{\prime} N \mid \mathscr{A}_{\mathscr{E}_{N} \mid}
$$


Next, for each $C \in \mathscr{A}_{N}$, there exist at least $\epsilon_{E} N$ choices for $y$ (by Lemma 8). Given $y$, there exist $K^{\prime}$ choices for $\phi$. Then $C$ and $\phi$ determine $\hat{C}$. Hence

$$
\text { (number of 4-tuples containing } C \text { ) } \geq K^{\prime} \epsilon_{E} N \quad \text { for each } C \in \mathscr{A}_{N} \text {, }
$$

which implies that

$$
\text { (number of 4-tuples) } \geq K^{\prime} \epsilon_{E} N\left|\mathscr{A}_{N}\right| \text {. }
$$

Part (a) of Proposition 7 now follows from (13) and (15).

(b) The right-hand inequality is trivial. To prepare for the proof of the left-hand inequality, fix $D$ as in Lemma 9 and let $D_{\text {Tile }}$ be the $d_{H}$-diameter of a tile (i.e. the largest value $d_{H}(u, v)$ where $u$ and $v$ are points in the same tile).

Let $\hat{C} \in \mathscr{A}_{\mathscr{E}_{N}}$. Since 0 is an extreme point of $\operatorname{conv}(\mathscr{N}(\hat{C})), 0$ must be a point of $\mathscr{N}(\hat{C})$. Therefore $d_{G}(0, \hat{C})<n_{\text {Tile }}$ (where $n_{\text {Tile }}$ is the number of vertices on one tile). Let $H_{1}$ be a closed (not necessarily lattice) half-space that has 0 on its boundary and contains $\operatorname{conv}(\mathscr{N}(\hat{C}))$. Let $H_{1}^{\perp}$ be the line through the origin that is perpendicular to the boundary of $H_{1}$, and let $\tilde{u}$ be the point on $H_{1}^{\perp}$ such that $d_{H}\left(\tilde{u}, H_{1}\right)=D+D_{\text {Tile }}$ (and hence $d_{H}(\tilde{u}, 0)=D+D_{\text {Tile }}$ also).

Let $u$ be an extreme point of the tile containing $\tilde{u}$. Then $d_{H}(u, 0) \geq d_{H}(\tilde{u}, 0)-d_{H}(\tilde{u}, u) \geq D$. By Lemma 9 , there exists a lattice-(hyper-)plane $L_{u}$ containing $u$ such that $L_{u} \cap H_{1}=\emptyset$. Let $H_{u}$ be the closed half-space with boundary $L_{u}$ that contains $H_{1}$. Observe that

$$
\begin{aligned}
d_{G}(u, \hat{C}) & \leq K_{G H} d_{H}(u, \hat{C}) \quad \text { (using Lemma 5) } \\
& \leq K_{G H}\left[d_{H}(u, \tilde{u})+d_{H}(\tilde{u}, 0)+d_{H}(0, \hat{C})\right] \\
& \leq K_{G H}\left(D+3 D_{\text {Tile }}\right) .
\end{aligned}
$$

So there exists a path $\pi_{1}$ (in $G$ ) from $\hat{C}$ to $u$ that is contained in $H_{u}$ and has at most $r$ edges, where $r=\left\lfloor K_{G H}\left(D+3 D_{\text {Tile }}\right)\right\rfloor$. Then $\hat{C} \cup \pi_{1}$ is an animal in $H_{u}$ with fewer than $N+r$ vertices. Finally, let $\pi_{2}$ be a path in $L_{u}$ sharing exactly one vertex with $\pi_{1}$ such that

$$
\left|\hat{C} \cup \pi_{1} \cup \pi_{2}\right|=N+r .
$$

For brevity, let $\pi$ denote the animal $\pi_{1} \cup \pi_{2}$. Let $\phi$ be an automorphism of the lattice that takes $u$ to 0 and $H_{u}$ to $\hat{H}_{0}$ (where the half-space $\hat{H}_{0}$ is specified in Definition 6). Let $C^{*}$ be the animal consisting of the image of $\hat{C} \cup \pi$ under $\phi$. Clearly, $C^{*} \in \mathscr{A} \mathscr{L}_{N+r}$. We claim that there is a constant $U_{L}$ (independent of $N$ and $C^{*}$ ) such that no more than $U_{L}$ different $\hat{C}$ 's in $\mathscr{A}_{\mathscr{E}_{N}}$ could give rise to the same $C^{*}$. To see this, observe that there is a bounded number of possible choices for $\phi(\pi)$ and $\phi(0)$ (the bound depends on $r$, but $r$ is fixed). Given $\phi(\pi)$ and $C^{*}$, we know $\phi(\hat{C})$; and given $\phi(0)$, there are exactly $K^{\prime}$ possible choices for $\phi$. Since $\hat{C}=\phi^{-1}(\phi(\hat{C}))$, this proves the claim, and part (b) follows.

Proof of Proposition 1; The boundedness of $a_{N}^{1 / N}$ (and hence of $t_{N}^{1 / N}$ ) follows from a general percolation argument, as described on pages 81-82 of Grimmett (1999).

We shall show the existence of the limit $\lambda_{a}$; the proof for $\lambda_{t}$ is essentially the same. Let $x_{A}$ and $x$ be vertices of $\mathscr{G}$ such that $x_{A}$ is a neighbour of the origin, $x_{A}$ is in the complement of $\hat{H}_{0}$ (from Definition (6), and $x_{A}$ is the midpoint of the line segment $\overline{0 x}$. We claim that there exists a latticehalf-space $\hat{H}_{x}$ which does not intersect $\hat{H}_{0}$ and whose boundary contains $x$ (and is perpendicular to 
$\overline{0 x})$. In $\mathscr{H}(5,3,4), \overline{0 x}$ must be perpendicular to the boundary plane of $\hat{H}_{0}$, so the boundary plane of $\hat{H}_{x}$ is the lattice plane containing $x$ that is perpendicular to $\overline{0 x}$. In $\mathscr{H}(f, v)$, it is not hard to see that $x$ is not in $\mathscr{N}\left(\hat{H}_{0}\right)$, so the existence of $\hat{H}_{x}$ follows from Lemma 3.2 of Madras and Wu (2005). Thus the claim holds for all choices of $\mathscr{G}$.

Let $\phi$ be an automorphism of $\mathscr{G}$ such that $\phi(0)=x$ and $\phi\left(\hat{H}_{0}\right)=\hat{H}_{x}$.

Consider positive integers $m$ and $n$. Let $C_{1}$ be an animal in $\mathscr{A} \mathscr{L}_{m}$ and let $C_{2}$ be an animal in $\mathscr{A} \mathscr{L}_{n}$. Then $\phi\left(C_{2}\right)$ is an animal contained in $\hat{H}_{x}$ that contains the vertex $x$. Let $C_{12}$ be the animal that is the union of $C_{1}, \phi\left(C_{2}\right)$, and $\overline{0 x}$. Then $C_{12} \in \mathscr{A}_{m+n+1}$. Moreover, since $\phi$ is fixed, any animal $C_{12}$ obtained in this way uniquely determines $C_{1}$ and $C_{2}$; that is, the mapping we have constructed from $\mathscr{A} \mathscr{L}_{m} \times \mathscr{A}_{\mathscr{L}_{n}}$ into $\mathscr{A}_{m+n+1}$ is clearly one-to-one. Therefore

$$
a_{m+n+1} \geq\left|\mathscr{A} \mathscr{L}_{m}\right|\left|\mathscr{A} \mathscr{L}_{n}\right|
$$

Combining this with Proposition 7 shows that

$$
a_{m+n+1} \geq\left(\frac{\epsilon_{E}}{U_{L}}\right)^{2} a_{m-r} a_{n-r} \quad \text { for all } m, n \geq r+1 .
$$

If we rewrite $i=m-r$ and $j=n-r$, and let $\tilde{a_{k}}=\left(\epsilon_{E} / U_{L}\right)^{2} a_{k}$, then (16) becomes

$$
\tilde{a}_{i+j+2 r+1} \geq \tilde{a}_{i} \tilde{a}_{j} \quad \text { for all } i, j \geq 1 \text {. }
$$

This is a variation on the usual super-multiplicative relation which holds for Euclidean lattice animals (e.g. see Section 6.1.1 of Janse van Rensburg 2000). This variation has been treated by Wilker and Whittington (1979), whose results imply our relations (1) and (2) for animals.

\section{Critical Behaviour of Lattice Animals and Lattice Trees}

The goal of this section is to prove Theorem 3. To this end, we begin by defining the half-space susceptibility $\chi_{A L}$,

$$
\chi_{A L}(z):=\sum_{N=1}^{\infty} N\left|\mathscr{A} \mathscr{L}_{N}\right| z^{N},
$$

which is the analogue of $\chi_{a}$ for animals in $\cup_{N} \mathscr{A} \mathscr{L}_{N}$ (recall Definitions 2 and 6). The proof of Theorem 3 relies on the following result, which says that $\chi_{A L}$ is within a constant factor of $\chi_{a}$. It is an immediate consequence of Proposition 7 .

Proposition 10. There exists a strictly positive constant $C_{A L}$ such that

$$
C_{A L} \chi_{a}(z) \leq \chi_{A L}(z) \leq \chi_{a}(z)
$$

for all $z \in\left(z_{a c} / 2, z_{a c}\right)$.

Proof of Theorem 3; The inequality

$$
K_{L}\left(z_{a c}-z\right)^{-1 / 2} \leq \chi_{a}(z)
$$


(for some constant $K_{L}$ ) is a known "mean field bound" that holds on fairly general lattices. Indeed, the proofs of Bovier et al. (1986) and Tasaki and Hara (1987) apply directly to hyperbolic lattices. The key is the establishment of the differential inequality

$$
\frac{d \chi_{a}(z)}{d z} \leq K \chi_{a}(z)^{3}
$$

(for some constant $K$ ); the integration of this inequality leads to the lower bound. We shall show that the reverse inequality holds in hyperbolic space, and that this leads to the desired upper bound.

Consider three mutually disjoint lattice-half-spaces $H_{1}, H_{2}$, and $H_{3}$, none of which contains the origin. For each $i=1,2,3$, let $\pi_{i}$ be a self-avoiding walk from the origin to a vertex $u_{i}$ on the boundary of $H_{i}$, such that $u_{i}$ is the only point of $H_{1} \cup H_{2} \cup H_{3}$ on $\pi_{i}$, and such that no two of the walks have any point in common besides the origin (see Figure 2). It is always possible to choose

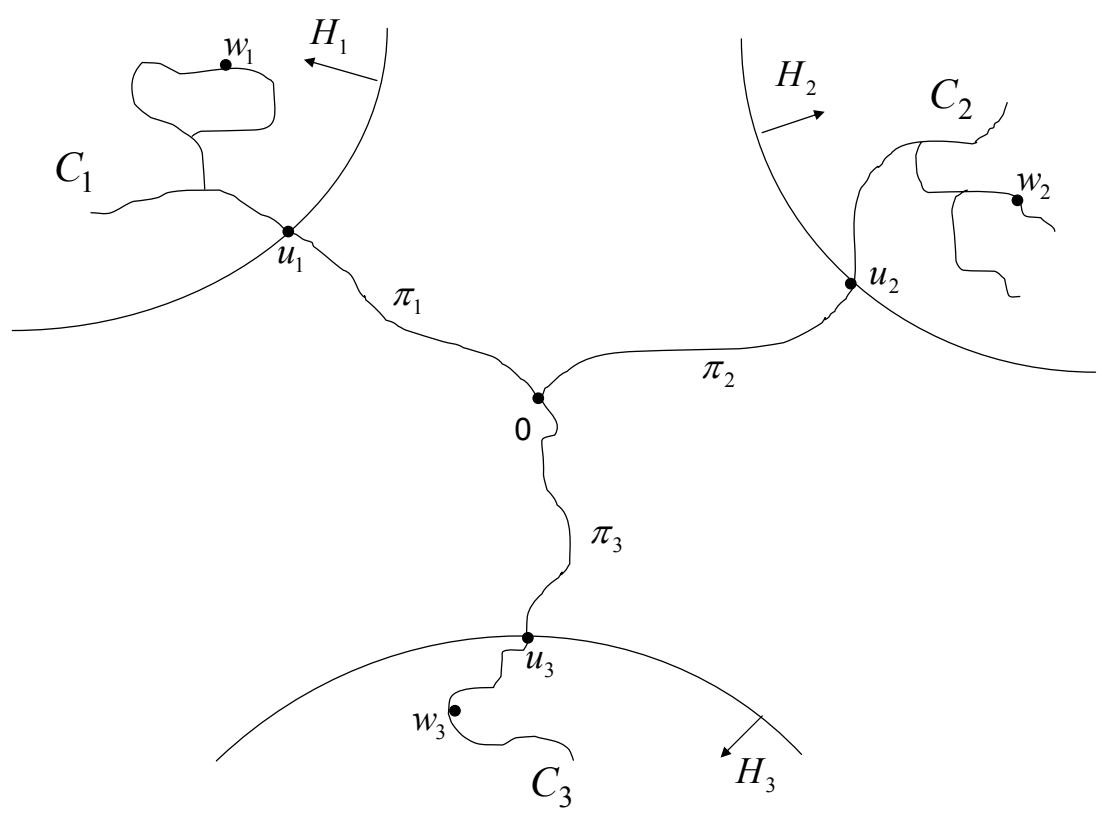

Figure 2: Proof of Theorem 3

the $H_{i}$ 's so that these $\pi_{i}$ 's exist; assume that we have done so. The $H_{i}$ 's and the $\pi_{i}$ 's will remain fixed for the rest of this proof.

For each $i=1,2,3$, let $\mathscr{A}[i]$ be the set of pairs $\left(C_{i}, w_{i}\right)$ where $C_{i}$ is an animal containing $u_{i}$ and contained in $H_{i}$, and $w_{i}$ is a vertex of $C_{i}$. Observe that

$$
\sum_{(C, w) \in \mathscr{A}[i]} z^{|C|}=\chi_{A L}(z) .
$$

Define the set $\mathscr{S}_{6}:=\mathscr{A}[1] \times \mathscr{A}[2] \times \mathscr{A}[3]$, consisting of 6-tuples $\left(C_{1}, w_{1}, C_{2}, w_{2}, C_{3}, w_{3}\right)$.

Before proceeding, we make the following graph-theoretical definition. Let $Q$ be a connected graph, and let $v_{1}, v_{2}$, and $v_{3}$ be vertices of $Q$ (not necessarily distinct). We say that the vertex $\hat{v}$ is a 
$\left(v_{1}, v_{2}, v_{3}\right)$-articulation point of $Q$ if $\hat{v}$ is on every path from $v_{1}$ to $v_{2}$, and every path from $v_{1}$ to $v_{3}$, and every path from $v_{2}$ to $v_{3}$. Note that a $\left(v_{1}, v_{2}, v_{3}\right)$-articulation point of $Q$ may not exist, but if it does exist then it is not hard to see that it must be unique.

Next, consider the set $\mathscr{S}_{3}$ of all triples $\left(D, y_{1}, y_{2}\right)$, where $D$ is an animal containing the origin, and $y_{1}$ and $y_{2}$ are vertices in $D$. Observe that

$$
\sum_{\left(D, y_{1}, y_{2}\right) \in \mathscr{S}_{3}} z^{|D|}=\sum_{N=1}^{\infty} N^{2} a_{N} z^{N}=z \frac{d \chi_{a}(z)}{d z} .
$$

Now, for any 6-tuple $\left(C_{1}, \ldots, w_{3}\right)$ in $\mathscr{S}_{6}$, let $\phi$ be an automorphism of $\mathscr{G}$ such that $\phi\left(w_{3}\right)=0$. If we now write

$$
y_{1}=\phi\left(w_{1}\right), y_{2}=\phi\left(w_{2}\right), \text { and } D=\phi\left(C_{1} \cup C_{2} \cup C_{3} \cup \pi_{1} \cup \pi_{2} \cup \pi_{3}\right),
$$

then $\left(D, y_{1}, y_{2}\right) \in \mathscr{S}_{3}$. By construction, we have

$$
|D|=\left|C_{1}\right|+\left|C_{2}\right|+\left|C_{3}\right|+q \text { where } q=\left|\pi_{1} \cup \pi_{2} \cup \pi_{3}\right|-3 .
$$

We claim that no triple $\left(D, y_{1}, y_{2}\right)$ in $\mathscr{S}_{3}$ could arise from more than $K^{\prime}$ 6-tuples in $\mathscr{S}_{6}$, where $K^{\prime}$ is the number of automorphisms of $\mathscr{G}$ that fix the origin. Indeed, for any 6-tuple $\left(C_{1}, \ldots, w_{3}\right)$ in $\mathscr{S}_{6}$, let $C^{\prime}$ be the animal $C_{1} \cup C_{2} \cup C_{3} \cup \pi_{1} \cup \pi_{2} \cup \pi_{3}$. Observe that the origin is the unique $\left(w_{1}, w_{2}, w_{3}\right)$ articulation point of $C^{\prime}$. (See Figure 2) If $D=\phi\left(C^{\prime}\right)$ for some automorphism $\phi$, then we know that there is a unique $\left(y_{1}, y_{2}, 0\right)$-articulation point of $D$, and that point must be $\phi(0)$. Thus there are only $K^{\prime}$ possible choices for the $\phi$ that transforms a 6-tuple into $\left(D, y_{1}, y_{2}\right)$. Since $\phi$ determines the 6-tuple (via $w_{1}=\phi^{-1}\left(y_{1}\right), w_{2}=\phi^{-1}\left(y_{2}\right), w_{3}=\phi^{-1}(0)$, and $C_{i}=H_{i} \cap \phi^{-1}(D)$ ), this proves the claim. Therefore

$$
\begin{aligned}
\frac{d \chi_{a}(z)}{d z} & =\frac{1}{z} \sum_{\left(D, y_{1}, y_{2}\right) \in \mathscr{S}_{3}} z^{|D|} \\
& \geq \frac{1}{z K^{\prime}} \sum_{\left(C_{1}, w_{1}, C_{2}, w_{2}, C_{3}, w_{3}\right) \in \mathscr{S}_{6}} z^{\left|C_{1}\right|+\left|C_{2}\right|+\left|C_{3}\right|+q} \\
& =\frac{z^{q-1}}{K^{\prime}} \prod_{i=1}^{3}\left(\sum_{\left(C_{i}, w_{i}\right) \in \mathscr{A}[i]} z^{\left|C_{i}\right|}\right) \\
& =\frac{z^{q-1}}{K^{\prime}} \chi_{A L}(z)^{3} \quad[\text { by Equation (21)] } \\
& \geq \frac{z^{q-1}\left(C_{A L}\right)^{3}}{K^{\prime}} \chi_{a}(z)^{3} \quad \text { [by Proposition[10]. }
\end{aligned}
$$

Thus we have proven the reverse inequality of (20), namely

$$
\frac{d \chi_{a}(z)}{d z} \geq K \chi_{a}(z)^{3} \quad \text { for all } z \in\left(z_{a c} / 2, z_{a c}\right) .
$$

Divide by $\chi_{a}(z)^{3}$ to obtain

$$
-\frac{d \chi_{a}(z)^{-2}}{d z} \geq 2 K \quad \text { for all } z \in\left(z_{a c} / 2, z_{a c}\right) .
$$


For $z_{a c} / 2<z_{1}<z_{2}<z_{a c}$, we integrate the above from $z_{1}$ to $z_{2}$ and obtain

$$
\chi_{a}\left(z_{1}\right)^{-2}-\chi_{a}^{-2}\left(z_{2}\right) \geq 2 K\left(z_{2}-z_{1}\right) .
$$

Now let $z_{2}$ increase to $z_{a c}$. Since $\chi_{a}\left(z_{2}\right)>0$, we obtain

$$
\chi_{a}\left(z_{1}\right)^{-2} \geq 2 K\left(z_{a c}-z_{1}\right) .
$$

Finally, take square roots of both sides of this inequality. Together with the bound (19), this completes the proof of (6).

\section{Critical Behaviour of Percolation}

We begin by adding some notation to what we introduced in Section 1.3 , following Grimmett (1999, Section 5.3) and Schonmann (2002). For $A, B \subset V(\mathscr{G})$, we write $\{A \leftrightarrow B\}$ to denote the event that there exists an occupied path from some point of $A$ to some point of $B$. If $A$ is a singleton $\{x\}$, then we simply write $\{x \leftrightarrow B\}$. Observe that $\{0 \leftrightarrow y\}=\{y \in \mathscr{C}(0)\}$. The complement of $\{A \leftrightarrow B\}$ is written $\{A \leftrightarrow B\}$. We also introduce a "ghost field" by saying that each site of $\mathscr{G}$ is painted green with probability $q$, independently of everything else. We write $P_{p, q}$ and $E_{p, q}$ for the probability measure and expectation in this enlarged probability space. Let $Q$ be the (random) set of green sites. Then the function $\theta(p, q)$ defined in Equation (7) may also be written as

$$
\theta(p, q)=P_{p, q}\{0 \longleftrightarrow Q\}
$$

We also introduce the modified susceptibility function

$$
\chi(p, q)=E_{p, q}(|\mathscr{C}(0)| ; \mathscr{C}(0) \cap Q=\emptyset\}=\sum_{y \in V} P_{p, q}\{y \in \mathscr{C}(0), 0 \leftrightarrow Q\} .
$$

To prove Theorem 4, we shall use the following lemmas from Schonmann (2002), applied to our hyperbolic graphs $\mathscr{G}$.

Lemma 11. Suppose there exist $\epsilon>0$ and $c>0$ and vertices $x_{1}, x_{2}$ such that for every $p \in\left(p_{c}-\epsilon, p_{c}\right)$,

$$
\sum_{y_{1}, y_{2} \in V} P_{p}\left\{x_{1} \leftrightarrow y_{1}, x_{2} \leftrightarrow y_{2}, x_{1} \leftrightarrow x_{2}\right\} \geq c(\chi(p))^{2} .
$$

Then $\gamma=1$ and $\Delta=2$.

Lemma 12. Suppose there exist $\epsilon>0$ and $c>0$ and vertices $u_{1}, u_{2}, u_{3}$ such that for every $p \in$ $\left(p_{c}-\epsilon, p_{c}\right)$ and every $q \in(0, \epsilon)$,

$$
\sum_{y \in V} P_{p, q}\left\{u_{1} \leftrightarrow y, u_{1} \leftrightarrow Q, u_{2} \leftrightarrow Q, u_{3} \leftrightarrow Q, u_{2} \leftrightarrow u_{3}\right\} \geq c \chi(p, q)(\theta(p, q))^{2} .
$$

Then $\beta=1$ and $\delta=2$.

The following lemma contains the key argument. It says that in the definition of susceptibility, we don't lose too much if we restrict our attention to the event that the origin lies on the boundary of (the convex hull of) its cluster. This is reasonable in hyperbolic space, where the boundary of a set is a nonvanishing fraction of the entire set. This lemma is an analogue of Proposition 10 , 
Lemma 13. Let $p_{1} \in\left(0, p_{c}\right)$. Let $\hat{H}_{0}$ be a lattice-half-space that has the origin on its boundary. Then there is an $\epsilon_{P}>0$ such that

$$
\epsilon_{P} \chi(p) \leq \sum_{y \in \hat{H}_{0}} P_{p}\left\{y \in \mathscr{C}(0), \mathscr{C}(0) \subset \hat{H}_{0}\right\}=\sum_{N=1}^{\infty} \sum_{C^{*} \in \mathscr{A} \mathscr{L}_{N}} N P_{p}\left\{\mathscr{C}(0)=C^{*}\right\}
$$

for every $p \in\left(p_{1}, p_{c}\right)$.

Proof: We use results for various classes of lattice animals, as developed in Proposition 7 . Any animal $C$ could be a cluster; that is, there is a non-zero probability that all edges in $C$ are occupied, and all edges incident to $C$ but not in $C$ are vacant. Let $\pi(C)$ be the probability that $C$ is a cluster. Observe that for any automorphism $\phi$ of $\mathscr{G}$ and any animal $C$, we have $\pi(C)=\pi(\phi(C))$.

We shall use the notation $\sum^{[N]}$ to denote the sum over all 4-tuples $(C, y, \hat{C}, \phi)$ constructed in the proof of part (a) of Proposition 7 , where $C \in \mathscr{A}_{N}$ and so on.

$$
\begin{array}{rlr}
\chi(p) & =\sum_{N=1}^{\infty} \sum_{C \in \mathscr{A}_{N}} N \pi(C) & \\
& \leq \sum^{[N]} \frac{1}{K^{\prime} \epsilon_{E}} \pi(C) & \text { [by Eq. (14)] }] \\
& =\sum^{[N]} \frac{1}{K^{\prime} \epsilon_{E}} \pi(\hat{C}) & {[\text { since } \pi(C)=\pi(\hat{C}) \text { when } C \text { and } \hat{C}} \\
& =\frac{1}{\epsilon_{E}} \sum_{N=1}^{\infty} \sum_{\hat{C} \in \mathscr{A}_{\mathscr{S}_{N}}} N \pi(\hat{C}) & \text { are in the same 4-tuple] }
\end{array}
$$

Next, recall the proof of part (b) of Proposition 7, There, a construction was defined for each cluster $\hat{C} \in \mathscr{A} \mathscr{E}_{N}$ that joined $\hat{C}$ to an animal with $r$ new vertices, and then applied a lattice automorphism, to obtain an animal $C^{*}$ in $\mathscr{A}_{\mathscr{L}_{N+r}}$ (where $r$ is a fixed constant). It was observed that no more than $U_{L}$ different $\hat{C}$ 's could give rise to the same $C^{*}$. Also, it is not hard to see that since $r$ is fixed, there is a constant $B$ such that $\pi(\hat{C}) \leq B \pi\left(C^{*}\right)$ for all $p \in\left(p_{1}, p_{c}\right)$. From this discussion, we see that

$$
\begin{aligned}
\frac{1}{\epsilon_{E}} \sum_{N=1}^{\infty} \sum_{\hat{C} \in \mathscr{A} \mathscr{E}_{N}} N \pi(\hat{C}) & \leq \frac{U_{L}}{\epsilon_{E}} \sum_{N=1}^{\infty} \sum_{C^{*} \in \mathscr{A} \mathscr{L}_{N+r}} N B \pi\left(C^{*}\right) \\
& \leq \frac{U_{L} B}{\epsilon_{E}} \sum_{M=1}^{\infty} \sum_{C^{*} \in \mathscr{A}_{M}} M \pi\left(C^{*}\right) .
\end{aligned}
$$

Combining this with the preceding inequalities proves the result, with $\epsilon_{P}=\epsilon_{E} /\left(U_{L} B\right)$.

Proof of Theorem 4 for $\gamma$ and $\Delta$ : We shall verify the hypotheses of Lemma 11, Let $L_{1}$ and $L_{2}$ be any two lattice-planes whose neighborhoods are disjoint [i.e. $\mathscr{N}\left(L_{1}\right) \cap \mathscr{N}\left(L_{2}\right)=\emptyset$ ], and let $x_{1}$ and $x_{2}$ be vertices such that $x_{1} \in L_{1}$ and $x_{2} \in L_{2}$. Let $H_{1}$ and $H_{2}$ be the two disjoint (closed) half-spaces whose boundaries are $L_{1}$ and $L_{2}$ respectively.

For $i=1,2$, and $y_{i} \in H_{i}$, we define the event

$$
D_{i}\left[y_{i}\right]=\left\{y_{i} \in \mathscr{C}\left(x_{i}\right), \mathscr{C}\left(x_{i}\right) \subset H_{i}\right\} .
$$


Then $D_{1}\left[y_{1}\right]$ is independent of $D_{2}\left[y_{2}\right]$, since $D_{i}\left[y_{i}\right]$ depends only on the states of the bonds in $H_{i} \cup \mathcal{N}\left(L_{i}\right)$. Let $p \in\left(p_{1}, p_{c}\right)$, where $p_{1}$ is chosen as in Lemma13, We have

$$
\begin{aligned}
& \sum_{y_{1}, y_{2} \in V} P_{p}\left\{x_{1} \leftrightarrow y_{1}, x_{2} \leftrightarrow y_{2}, x_{1} \leftrightarrow x_{2}\right\} \\
& \geq \sum_{y_{1} \in H_{1}, y_{2} \in H_{2}} P_{p}\left(D_{1}\left[y_{1}\right] \cap D_{2}\left[y_{2}\right]\right) \\
& =\sum_{y_{1} \in H_{1}, y_{2} \in H_{2}} P_{p}\left(D_{1}\left[y_{1}\right]\right) P_{p}\left(D_{2}\left[y_{2}\right]\right) \\
& \geq\left(\epsilon_{P} \chi(p)\right)^{2} \quad \text { [by Lemma[13]. }
\end{aligned}
$$

This proves (24), and hence we have shown that $\gamma=1$ and $\Delta=2$.

Now we prepare for Lemma 12, We begin with the following analogue of Lemma 13 ,

Lemma 14. Let $p_{1} \in\left(0, p_{c}\right)$ and let $q_{1} \in(0,1)$. Let $H$ be a lattice-half-space whose boundary contains the origin. Then there exist constants $\epsilon_{Q}>0$ and $\epsilon_{\theta}>0$ such that

$$
\epsilon_{Q} \chi(p, q) \leq \sum_{y \in H} P_{p, q}\{y \in \mathscr{C}(0), \mathscr{C}(0) \subset H, 0 \leftrightarrow Q\}
$$

and

$$
\epsilon_{\theta} \theta(p, q) \leq P_{p, q}\{\mathscr{C}(0) \subset H, 0 \leftrightarrow Q\}
$$

for every $p \in\left(p_{1}, p_{c}\right)$ and $q \in\left(0, q_{1}\right)$.

Proof: Most of the work has already been done in the proof of Lemma 13, First, we have

$$
\begin{aligned}
\chi(p, q) & =\sum_{N=1}^{\infty} \sum_{C \in \mathscr{A}_{N}} N \pi(C)(1-q)^{N} \\
& \leq \frac{U_{L} B}{\epsilon_{E}} \sum_{N=1}^{\infty} \sum_{C^{*} \in \mathscr{A} \mathscr{L}_{N+r}} N \pi\left(C^{*}\right)(1-q)^{N} \\
& \leq \frac{U_{L} B}{\epsilon_{E}} \sum_{M=1}^{\infty} \sum_{C^{*} \in \mathscr{A} \mathscr{L}_{M}} M \pi\left(C^{*}\right)(1-q)^{M}(1-q)^{-r} .
\end{aligned}
$$

Therefore Equation (27) holds with $\epsilon_{Q}=\left(1-q_{1}\right)^{r} \epsilon_{E} / U_{L} B$.

Similarly, we have

$$
\begin{aligned}
\theta(p, q) & =\sum_{N=1}^{\infty} \sum_{C \in \mathscr{A}_{N}} N \pi(C)\left(\frac{1-(1-q)^{N}}{N}\right) \\
& \leq \frac{U_{L} B}{\epsilon_{E}} \sum_{N=1}^{\infty} \sum_{C^{*} \in \mathscr{A} \mathscr{L}_{N+r}} N \pi\left(C^{*}\right)\left(\frac{1-(1-q)^{N}}{N}\right) \\
& \leq \frac{U_{L} B}{\epsilon_{E}} \sum_{M=1}^{\infty} \sum_{C^{*} \in \mathscr{A} \mathscr{L}_{M}} \pi\left(C^{*}\right)\left(1-(1-q)^{M}\right)
\end{aligned}
$$

[since $1-(1-q)^{N} \leq 1-(1-q)^{N+r}$ ]. 
This proves Equation (28).

We are now ready to complete the proof of Theorem 4.

Proof of Theorem 4 for $\beta$ and $\delta$ : We shall verify the hypotheses of Lemma 12, Similarly to the proof of Theorem 3, let $H_{1}, H_{2}$, and $H_{3}$ be three lattice-half-spaces whose neighbourhoods are pairwise disjoint [i.e. $\mathcal{N}\left(H_{j}\right) \cap \mathscr{N}\left(H_{k}\right)=\emptyset$ for $1 \leq j<k \leq 3$ ]. Let $u_{1}, u_{2}$ and $u_{3}$ be vertices such that, for each $i, u_{i}$ is contained in the boundary plane of $H_{i}$.

For $y \in H_{1}$, we define the event

$$
E_{1}[y]=\left\{y \in \mathscr{C}\left(u_{1}\right), \mathscr{C}\left(u_{1}\right) \subset H_{1}, u_{1} \leftrightarrow Q\right\} .
$$

For $i=2,3$, define the events

$$
E_{i}=\left\{\mathscr{C}\left(u_{i}\right) \subset H_{i}, u_{i} \longleftrightarrow Q\right\} .
$$

Then, for any $y \in H_{1}$, the three events $E_{1}[y], E_{2}$, and $E_{3}$ are independent, since they depend only on events in $\mathscr{N}\left(H_{1}\right), \mathscr{N}\left(H_{2}\right)$, and $\mathscr{N}\left(H_{3}\right)$ respectively. For $p$ and $q$ as in Lemma 14, we have

$$
\begin{array}{ll}
\sum_{y \in V} P_{p, q}\left\{u_{1} \leftrightarrow y, u_{1} \leftrightarrow Q, u_{2} \leftrightarrow Q, u_{3} \leftrightarrow Q, u_{2} \leftrightarrow u_{3}\right\} \\
\geq \sum_{y \in H_{1}} P_{p, q}\left(E_{1}[y] \cap E_{2} \cap E_{3}\right) \\
=\sum_{y \in H_{1}} P_{p, q}\left(E_{1}[y]\right) P_{p, q}\left(E_{2}\right) P_{p, q}\left(E_{3}\right) \\
\geq \epsilon_{Q} \chi(p, q)\left(\epsilon_{\theta} \theta(p, q)\right)^{2}
\end{array}
$$

This proves (25), and hence we have shown that $\beta=1$ and $\delta=2$.

\section{Acknowledgements}

We are grateful to Karoly Bezdek, Ragnar-Olaf Buchweitz, Asia Weiss, and Walter Whiteley for helpful discussions about hyperbolic geometry, and to a referee for helpful comments.

\section{References}

[1] Benjamini, I., Lyons, R., Peres, Y. and Schramm, O. (1999). Critical percolation on any nonamenable group has no infinite clusters. Ann. Probab. 27, 1347-1356. MR1733151

[2] Benjamini, I. and Schramm, O. (1996). Percolation beyond $\mathbf{Z}^{d}$, many questions and a few answers. Electronic Commun. Probab. 1, Paper no. 8, 71-82. MR1423907 (97j:60179)

[3] Benjamini, I. and Schramm, O. (2001). Percolation in the hyperbolic plane. J. Amer. Math. Soc. 14, 487-507. MR1815220 (2002h:82049) 
[4] Borgs, C., Chayes, J., van der Hofstad, R., and Slade, G. (1999). Mean-field lattice trees. Ann. Combin. 3, 205-221. MR1772346 (2001i:82036)

[5] Bourdon, M. (1993). Actions quasi-convexes d'un groupe hyperbolique, flot géodésique. Thesis, Orsay.

[6] Bovier, A., Fröhlich, J., and Glaus, U. (1986). Branched polymers and dimensional reduction. In Critical Phenomena, Random Systems, and Gauge Theories, K. Osterwalder and R. Stora, eds. North-Holland, Amsterdam. MR0880538 (88h:82057)

[7] Bowen, R. and Series, C. (1979). Markov maps associated with Fuchsian groups. Inst. Hautes Etudes Sci. Publ. Math. 50, 153-170. MR0556585 (81b:58026)

[8] Brydges, D.C. and Imbrie, J.Z. (2003). Branched polymers and dimensional reduction. Ann. Math. 158, 1019-1039. MR2031859 (2005c:82051)

[9] Coxeter, H.S.M. (1956). Regular honeycombs in hyperbolic space. In Proceedings of the International Congress of Mathematicians, Amsterdam, 1954, Vol. III, 155-169. North-Holland, Amsterdam. (Reprinted in Coxeter, H.S.M. (1968), Twelve Geometric Essays, Southern Illinois University Press, Carbondale.) MR0087114 (19,304c)

[10] Coxeter, H.S.M. (1963). Regular Polytopes. Second edition. Macmillan, New York. MR0151873 (27 \#1856)

[11] Grimmett, G. (1999). Percolation. Second Edition. Springer, Berlin and Heidelberg. MR1707339 (2001a:60114)

[12] Grimmett, G.R. and Newman, C.M. (1990). Percolation in $\infty+1$ dimensions. In Disorder in Physical Systems. G. R. Grimmett and D. J. A. Welsh, eds., Clarendon Press, Oxford, 167-190. MR1064560 (92a:60207)

[13] Grünbaum, B. (2003). Convex Polytopes. Second edition. Springer-Verlag, New York. MR1976856 (2004b:52001)

[14] Haagerup, U. and Munkholm, H.J. (1981). Simplices of maximal volume in hyperbolic $n$ space. Acta Math. 147, 1-11. MR0631085 (82j:53116)

[15] Häggström, O. and Jonasson, J. (2006). Uniqueness and non-uniqueness in percolation theory. Probab. Surv. 3, 289-344. MR2280297 (2007m:60297)

[16] Iversen, B. (1992). Hyperbolic Geometry. Cambridge University Press, Cambridge. MR1205776 (94b:51023)

[17] Janse van Rensburg, E.J. (2000). The Statistical Mechanics of Interacting Walks, Polygons, Animals and Vesicles. Oxford University Press, Oxford. MR1858028 (2003a:82032)

[18] Jonasson, J. and Steif, J. (1999). Amenability and phase transition in the Ising model. J. Theor. Probab. 12, 549-559. MR1684757 (2000b:60238) MR1684757 (2000b:60238)

[19] Klarner, D.A. (1967). Cell growth problems. Canad. J. Math. 19, 851-863. MR0214489 (35 \#5339) 
[20] Klein, D.J. (1981). Rigorous results for branched polymer models with excluded volume. $J$. Chem. Phys. 75, 5186-5189.

[21] Lalley, S.P. (1998). Percolation on Fuchsian groups. Ann. Inst. Henri Poincaré 34, 151-177. MR1614583 (99g:60190)

[22] Lalley, S.P. (2001). Percolation clusters in hyperbolic tessellations. Geom. Funct. Anal. 11, 9711030. MR1873136 (2002i:60183)

[23] Lubensky, T.C. and Isaacson, J. (1979). Statistics of lattice animals and dilute branched polymers. Phys. Rev. A 20, 2130-2146.

[24] Madras, N. (1995). A rigorous bound on the critical exponent for the number of lattice trees, animals, and polygons. J. Statist. Phys. 78, 681-699. MR1315231 (95m:82076)

[25] Madras, N. and Wu, C.C. (2005). Self-avoiding walks in hyperbolic graphs. Combin. Probab. Comput. 14, 523-548. MR2160417 (2006e:60061)

[26] Otter, R. (1948). The number of trees. Ann. Math. 49, 583-599. MR0025715 (10,53c)

[27] Pak, I. and Smirnova-Nagnibeda, T. (2000). On non-uniqueness of percolation on nonamenable Cayley graphs. C.R. Acad. Sci. Paris Sér. I Math. 330, 495-500. MR1756965 (2000m:60116)

[28] Parisi, G. and Sourlas, N. (1981). Critical behavior of branched polymers and the Lee-Yang edge singularity. Phys. Rev. Lett. 46, 871-874. MR0609853 (82d:82058)

[29] Pólya, G. (1937). Kombinatorische Anzahlbestimmungen für Gruppen, Graphen, und chemische Verbindungen. Acta Math. 68, 145-254.

[30] Pólya, G. and Read, R.C. (1987). Combinatorial Enumeration of Groups, Graphs, and Chemical Compounds. Springer-Verlag, New York. MR0884155 (89f:05013)

[31] Ratcliffe, J.G. (1994). Foundations of Hyperbolic Manifolds. Springer-Verlag, New York. MR1299730 (95j:57011)

[32] Rietman, R., Nienhuis, B and Oitmaa, J. (1992). The Ising model on hyperlattices. J. Phys. A.: Math. Gen. 25, 6577-6592. MR1210879 (94e:82026)

[33] Schonmann, R. H. (2001). Multiplicity of phase transitions and mean-field criticality on highly non-amenable graphs. Commun. Math. Phys. 219, 271-322. MR1833805 (2002h:82036)

[34] Schonmann, R.H. (2002). Mean-field criticality for percolation on planar non-amenable graphs. Commun. Math. Phys. 225, 453-463. MR1888869 (2003m:82039)

[35] Slade, G. (2006). The Lace Expansion and its Applications. Ecole d'Eté de Probabilités de SaintFlour XXXIV-2004. Lecture Notes in Mathematics v. 1879. Springer, Berlin and Heidelberg. MR2239599 (2007m:60301)

[36] Swierczak, E. and Guttmann, A. J. (1996). Self-avoiding walks and polygons on non-Euclidean lattices. J. Phys. A.: Math. Gen. 25, 7485-7500. MR1425834 (98a:82058) 
[37] Tasaki, H. and Hara, T. (1987). Critical Behaviour in a System of Branched Polymers. Prog. Theor. Phys. Suppl. 92, 14-25. MR0934664 (89f:82054)

[38] Tykesson, J. (2007). The number of unbounded components in the Poisson Boolean model of continuum percolation in hyperbolic space. Electron. J. Probab. 12, 1379-1401. MR2354162 (2008k:60248)

[39] Vanderzande, C. (1998). Lattice Models of Polymers. Cambridge Lecture Notes in Physics, v. 11. Cambridge University Press, Cambridge.

[40] Wilker, J.B. and Whittington, S.G. (1979). Extension of a theorem on super-multiplicative functions. J. Phys. A: Math. Gen. 12, L245-247. MR0545387 (81c:26019)

[41] Wu, C.C. (2000). Ising models on hyperbolic graphs II. J. Statist. Phys. 100, 893-904. MR1798548 (2002h:82027) 